\title{
Factors related to suicide attempts among individuals with major depressive disorder
}

This article was published in the following Dove Press journal:

International Journal of General Medicine

10 April 2012

Number of times this article has been viewed

\author{
Chidchanok Ruengorn ${ }^{1,2}$ \\ Kittipong Sanichwankul ${ }^{3}$ \\ Wirat Niwatananun ${ }^{2}$ \\ Suwat Mahatnirunkul ${ }^{3}$ \\ Wanida Pumpaisalchai ${ }^{3}$ \\ Jayanton Patumanond' \\ 'Clinical Epidemiology Unit, Faculty \\ of Medicine, ${ }^{2}$ Department of \\ Pharmaceutical Care, Faculty of \\ Pharmacy, Chiang Mai University, \\ Chiang Mai, Thailand; '3Suanprung \\ Psychiatric Hospital, Chiang Mai, \\ Thailand
}

Correspondence: Jayanton Patumanond Clinical Epidemiology Unit, Faculty of Medicine, Chiang Mai University, Suthep Rd, Muang District,

Chiang Mai 50200, Thailand

Tel +6653946306

Fax +66 53945476

Email j.patumanond@yahoo.com
Background: Major depressive disorder (MDD) is the leading cause of suicidal behaviors. Risk related to suicide attempts among individuals with MDD remains uninvestigated in upper northern Thailand, where the completed suicide rate is the highest in the nation.

Objective: To examine risk related to suicide attempts among individuals with MDD.

Methods: Individuals diagnosed with MDD using the International Statistical Classification of Diseases and Related Health Problems 10th Revision (ICD-10), codes F32.x and F33.x, seeking care at Suanprung Psychiatric Hospital between October 2006 and May 2009 were eligible. All individuals with MDD admitted due to suicide attempts were defined as cases ( $n=186$ ), and four controls per case were selected from those who did not attempt suicide on the same day or within a week of case selection $(n=914)$. Their medical charts were reviewed for sociodemographic and clinical factors influencing suicide attempts using multivariable logistic regression analysis.

Results: Factors related to suicide attempts were stressful life events (adjusted odds ratio [OR], 2.32; 95\% confidence interval [CI]: 1.27-4.24), alcohol use (adjusted OR, 2.08; 95\% CI: 1.29-3.34), intermittent or poor psychiatric medications adherence (adjusted OR, 2.25; 95\% CI: 1.44-3.51), up to two previous suicide attempts (adjusted OR, 3.64; 95\% CI: 2.32-5.71), more than two previous suicide attempts (adjusted OR, 11.47; 95\% CI: 5.73-22.95), and prescribed antipsychotics (adjusted OR, 3.84; 95\% CI: 2.48-5.95). Risk factors that were inversely related to suicide attempts were increasing years of MDD treatment; one to five years (adjusted OR, 0.22; 95\% CI: 0.11-0.44), over five years (adjusted OR, 0.44; 95\% CI: 0.23-0.86), and antidepressant prescribed (norepinephrine [NE] and/or serotonin reuptake inhibitors [SRIs], adjusted OR, 0.28; 95\% CI: $0.10-0.78$ ). The final model explained $85.8 \%$ probability of suicide attempts.

Conclusion: Seven key factors suggested from this study may facilitate clinicians to identify individuals with MDD at risk of suicide attempt and provide them close monitoring, timely assessment, and intensive treatments.

Keywords: mood disorders, affective disorders, suicide behaviors, risk factors

\section{Introduction}

It is expected that major depressive disorder (MDD) will be the second-highest cause of global morbidity and mortality by $2020 .{ }^{1}$ Suicide is the primary reason for increased mortality in depression, with a general standard mortality ratio (SMR) of $20.4 \%{ }^{2}$ Suicidal ideation is highly prevalent (58\%) among those with MDD, and appears to be a precondition for suicide attempts. Suicide attempts are strongly predictive of further suicide attempts and completion of suicide. ${ }^{3}$ Between $20 \%$ and $65 \%$ of those with MDD who died by committing suicide have had a history of previous 
suicide attempts. ${ }^{4}$ The understanding of the characteristics related to suicide attempts in those with MDD is important to identify the individuals at high risk and may be beneficial in preventing future suicide.

Several previous studies have reported factors that are predictors of suicide attempts as being younger age, ${ }^{3}$ low perceived social support and occupational functioning, ${ }^{3,5}$ low level of education, ${ }^{6}$ lack of a partner, ${ }^{5,7}$ severity or duration of depression, ${ }^{3,8}$ time spent in a major depressive episode or in partial remission, ${ }^{5,7}$ smoking, ${ }^{9}$ current alcohol dependence or substance abuse, ${ }^{1,3,6,10}$ suicidal ideation, ${ }^{3,8,11}$ previous suicide attempts,,${ }^{5,712}$ negative life events, ${ }^{6,11}$ hopelessness, ${ }^{13,14}$ family history of suicide, ${ }^{14}$ aggression, impulsivity, ${ }^{8,15}$ and hostility. ${ }^{8}$ However, causation of suicide attempts in MDD is complex, and it may not be applicable to generalize the predictive factors identified in previous research because of discrepancies in population and methodology.

To date, the risk factors related to suicide attempts in individuals with MDD in upper northern Thailand remain uninvestigated. This area is known to have the highest incidence rate of completed suicides in the country; 12.5 compared to 5.90 per 100,000 of the nationwide population in $2010 .{ }^{16}$ The suicide attempt rate was also markedly high: 34.1 per 100,000 people. This study explored the risk factors related to suicide attempts in individuals with MDD.

\section{Materials and methods}

\section{Study setting}

Suanprung Psychiatric Hospital, a large university-affiliated, 700-bed, referral tertiary psychiatric hospital, located in Chiang Mai, has provided medical care to people in the upper northern region of Thailand since 1938. Each year, over 6000 and 60,000 services are given to inpatients and outpatients, respectively, at this hospital. Among the high burden of psychiatric illnesses treated by the hospital, MDD has always ranked in the top five. In the consecutive years from 2009 to 2011, the numbers of MDD inpatients were 306,344 , and 270, respectively. A total of 6412, 6687, and 6980 outpatient visits were made by individuals with MDD in the corresponding years, respectively. ${ }^{17}$

\section{Study population}

Eligible participants were individuals with MDD who had been diagnosed by psychiatrists and coded using the International Statistical Classification of Diseases and Related Health Problems 10th Revision (ICD-10): ICD-10 F32.x and F33.x. All participants were those who had been admitted due to suicide attempts, regardless of the number of attempt(s) $(n=186)$, and four controls per case were selected from MDD inpatients outpatients who did not attempt suicide on the same day first, then within a week of case selection $(n=914)$. That visit date was set as an index date. A simple random sampling method was used to select controls from the hospital database if possible numbers exceeded four. The medical files of inpatient and outpatient visits between October 2006 and May 2009 were reviewed.

\section{Procedures and data collection}

Information from medical charts was extracted by five trained nursing staff at the hospital. Prior to data collection, two meetings of the researchers were held to discuss and clarify a definition, and measures, of each variable.

Sociodemographic and clinical factors at the index date were collected and were then selected and categorized based on both a review of the literature and on the opinion of the psychiatrists in the research team. The sociodemographic factors that were included were: sex; age; marital status; educational level; occupation; religion; living status (alone or with others); having children; and stressful life events such as interpersonal relationship problems, loss of loved ones, and economic crisis prior to, or at, the index date. Social support was divided into good or excellent, moderate, and little or very little.

Clinical factors included were ICD-10 groups categorizing participants into those with mild-to-moderate depression (coded F32.0, 32.1, 33.0, 33.1), severe depression (coded F32.2, 33.2), or psychosis (coded F32.3, 33.3). Duration of MDD treatment was defined as year(s) from MDD treatment initiation to the index date. Previous admission (yes/no) was psychiatric hospitalization before the index time. Number of previous suicide attempt(s) and previous suicidal ideation (yes/no) were retrieved. Psychiatric symptoms presented at the index time were hallucination, hearing voices, or other psychotic symptoms. Psychotic comorbidity was taken from recorded ICD-10 codes and grouped into alcohol/substance dependence, or others. Somatic illness was any chronic diseases. Smoking, alcohol use, and any substance abuse were behaviors at the index time.

Medication prescriptions redeemed were categorized by their mechanism of action and evidence of association with suicide attempts. Antidepressants were grouped as norepinephrine (NE) and/or serotonin reuptake inhibitors (SRIs), such as amitriptyline, nortriptyline, venlafaxine, and selective serotonin reuptake inhibitors (SSRIs), such as fluoxetine and fluvoxamine. Antipsychotics (yes/ no) included zuclopenthixol decanoate, haloperidol, 
clozapine, risperidone, and olanzapine. Anxiolytics consisted of benzodiazepine, and other anxiolytics, including chlordiazepoxide. Pharmacists asked participants/relatives questions around their medication-taking behavior before the index time in order to assess adherence to psychiatric medication. Adherence was classified as "good or excellent," meaning "always took medication or rarely missed any dose," while "intermittently and poorly adhere" meant "took medication off and on, or did not take medication at least a week before an index date."

Other factors included were family history of mental disorders, and family history for attempted or completed suicide, method(s) used for suicide attempts, and numbers of admission days. Two institutional review boards, at The Faculty of Medicine at Chiang Mai University, and Suanprung Psychiatric Hospital, approved the study protocol.

\section{Statistical analyses}

Data were analyzed in two steps. In the first of these, sociodemographic and clinical indicators related to suicide attempts were investigated using univariable logistic regression. Those giving $P<0.10$ were selected for the second step. The set of characteristics independently related to suicide attempts were finally determined using multivariable stepwise logistic regression. Missing values for risk factors were handled by a multiple imputation method (MI) using logistic regression. ${ }^{18}$ The cut-off points of variables were determined by clinical significance, and statistical significance using the prediction probability by the receiver operating characteristic curve (ROC) value. Statistical significance level was set at 0.05 .

Sample size calculation was based on proportions of suicide attempts and non-attempts among those with MDD taken from previous studies. ${ }^{5,8,19}$ Two different proportions, that is, $58.2 \%$ of suicide attempters receiving antipsychotics and $39.8 \%$ of nonattempters receiving antipsychotics, were used. ${ }^{19}$ The total number of participants with MDD admitted with suicide attempts during the study period was 186 , so the required number of controls was calculated. Four controls per case, or 744 people, were enough to ensure a power of $90 \%$ and 0.05 type I error. When adding $20 \%$ of people to compensate for missing data, 893 controls were needed. We used STATA $^{\circledR} 11.0$ (StataCorp LP; College Station, TX) to perform all statistical analyses.

\section{Results}

Among the 1100 individuals with MDD included in the final analysis, $186(16.9 \%)$ attempted suicide. Of these, $151(81.6 \%)$ used one method, $24(13.0 \%)$ used two methods, and eight (4.2\%) used more than two methods. Drug overdose was the most common method used in 145 (78.0\%) people, followed by hanging ( $\mathrm{n}=123 ; 66.1 \%)$, cutting with a knife or sharp object $(n=66 ; 35.5 \%)$, swallowing pesticide $(n=60 ; 32.2 \%)$, jumping from a high place $(n=21 ; 11.3 \%)$, jumping into water $(n=10 ; 5.4 \%)$, and using firearms $(\mathrm{n}=3 ; 1.6 \%)$.

According to univariable analysis, those who attempted suicide were more likely to be male, younger, single, have a lower level of education, be unemployed, have no children, have less social support, and be experiencing stressful life events (Table 1).

They also presented with psychosis, alcohol/substance dependence, less duration of MDD ( $<1$ years), previous psychiatric hospitalization, had psychotic symptoms at the index time, had a family history for attempted or completed suicide, had previously attempted suicide, had previously had suicidal ideation, were not adherent to medication, and had been prescribed antipsychotics. Antidepressants had a protective effect against suicide attempts (Table 2).

Factors related to suicide attempts were stressful life events (adjusted odds ratio [OR], 2.32; 95\% confidence interval [CI]: 1.27-4.24), alcohol use (adjusted OR, 2.08; 95\% CI: 1.29-3.34), intermittent or poor psychiatric medication adherence (adjusted OR, 2.25; 95\% CI: 1.44-3.51), up to two previous suicide attempts (adjusted OR, 3.64; 95\% CI: 2.32 5.71), more than two previous suicide attempts (adjusted OR, 11.47; 95\% CI: 5.73-22.95), and prescribed antipsychotics (adjusted OR, 3.84; 95\% CI: 2.48-5.95). Risk factors that were inversely related to suicide attempts were increasing years of MDD treatment; one to five years (adjusted OR, 0.22; 95\% CI: 0.11-0.44), over five years (adjusted OR, 0.44; 95\% CI: 0.23-0.86), and antidepressant prescribed (norepinephrine [NE] and/or serotonin-reuptake inhibitors [SRIs]) (adjusted OR, 0.28; 95\% CI: 0.10-0.78) (Table 3). The final model explained $85.8 \%$ of the probability of suicide attempts, based on the area under the ROC curve.

\section{Discussion}

The present study revealed seven sociodemographic and clinical factors that were statistically significantly associated with suicide attempts among individuals with MDD.

Stressful life events posed a twofold increase in risk of suicide attempts in our study. Stressful life events, particularly family or interpersonal relationship problems, have been documented in another study conducted in Thailand that examined the relationship with suicide attempts. ${ }^{6,11}$ It was found that stressful life events triggered an elevation in 
Table I Sociodemographic factors of patients with MDD classified by suicide attempts

\begin{tabular}{|c|c|c|c|c|}
\hline \multirow{2}{*}{$\begin{array}{l}\text { Sociodemographic } \\
\text { factors }\end{array}$} & \multicolumn{2}{|c|}{ Suicide attempts } & \multirow[t]{2}{*}{ Crude OR $(95 \% \mathrm{CI})$} & \multirow[t]{2}{*}{$P$-value } \\
\hline & $\begin{array}{l}\text { Yes } \\
(n=186)\end{array}$ & $\begin{array}{l}\text { No } \\
(n=9 \mid 4)\end{array}$ & & \\
\hline Sex & & & & 0.037 \\
\hline Male & $72(20.3)$ & $282(79.7)$ & 1.00 & \\
\hline Female & $114(15.3)$ & $632(84.7)$ & $0.7 \mathrm{I}(0.5 \mathrm{I}-0.98)$ & \\
\hline \multicolumn{5}{|l|}{ Age (years) } \\
\hline $13-29$ & $44(24.0)$ & $139(76.0)$ & 1.00 & \\
\hline $30-39$ & $50(23.5)$ & $163(76.5)$ & $0.97(0.61-1.54)$ & 0.894 \\
\hline $40-49$ & $45(\mid 7.0)$ & $220(83.0)$ & $0.65(0.40-1.03)$ & 0.067 \\
\hline $50-59$ & $28(9.7)$ & $26 I(90.3)$ & $0.34(0.20-0.57)$ & $<0.001$ \\
\hline$\geq 60$ & $17(12.4)$ & $120(87.6)$ & $0.48(0.24-0.82)$ & 0.010 \\
\hline \multicolumn{5}{|l|}{ Education } \\
\hline No or primary school & $89(15.6)$ & $481(84.4)$ & 1.00 & \\
\hline Junior to high school & $57(24.2)$ & I 78 (75.7) & $1.73(1.19-2.5 \mathrm{I})$ & 0.004 \\
\hline Diploma & $13(18.6)$ & $57(81.4)$ & $1.23(0.65-2.35)$ & 0.524 \\
\hline Bachelor or higher & $13(6.9)$ & $176(93.1)$ & $0.40(0.22-0.73)$ & 0.003 \\
\hline Marital status & & & & 0.028 \\
\hline Single & $5 \mathrm{I}(20.7)$ & $195(79.3)$ & 1.00 & \\
\hline Married & $101(15.1)$ & $567(84.9)$ & $0.68(0.47-0.99)$ & 0.044 \\
\hline Widowed/divorced or others & $33(19.5)$ & $136(80.5)$ & $0.93(0.57-\mid .5 I)$ & 0.764 \\
\hline \multicolumn{5}{|l|}{ Occupation } \\
\hline Government or private employee & $77(16.8)$ & $381(83.2)$ & 1.00 & \\
\hline Laborer & $40(16.0)$ & $210(84.0)$ & $0.94(0.62-1.43)$ & $0.78 \mathrm{I}$ \\
\hline Housewife/student & $25(10.5)$ & $214(89.5)$ & $0.58(0.36-0.94)$ & 0.026 \\
\hline Unemployed & $4 \mathrm{I}(29.5)$ & $98(70.5)$ & $2.07(1.33-3.21)$ & 0.001 \\
\hline \multicolumn{5}{|l|}{ Religion } \\
\hline Buddhism & $|7|(\mid 6.6)$ & $857(83.4)$ & 1.00 & \\
\hline Others & $8(16.7)$ & $40(83.3)$ & $1.00(0.46-2.18)$ & 0.995 \\
\hline \multicolumn{5}{|l|}{ Number of children } \\
\hline None & $32(32.3)$ & $67(67.7)$ & 1.00 & \\
\hline $\mathrm{I}-2$ & $51(15.4)$ & $281(84.6)$ & $0.38(0.23-0.64)$ & $<0.00$ I \\
\hline$>2$ & $16(15.1)$ & $90(84.9)$ & $0.37(0.1-0.73)$ & 0.004 \\
\hline \multicolumn{5}{|l|}{ Living alone } \\
\hline No & $161(17.0)$ & $786(83.0)$ & 1.00 & \\
\hline Yes & $12(19.4)$ & $50(80.6)$ & $\mathrm{I} .17(0.6 \mathrm{I}-2.25)$ & 0.634 \\
\hline \multicolumn{5}{|l|}{ Social support } \\
\hline Good or excellent & $88(13.6)$ & $560(86.4)$ & 1.00 & \\
\hline Moderate & $52(21.2)$ & $193(78.8)$ & I.7I (I.|7-2.5I) & 0.005 \\
\hline Little or very little & $24(28.2)$ & $61(71.8)$ & $2.50(1.48-4.22)$ & 0.001 \\
\hline \multicolumn{5}{|l|}{ Stressful life events } \\
\hline No & $24(8.3)$ & 267 (9l.8) & 1.00 & \\
\hline Yes & $162(20.0)$ & $647(80.0)$ & $2.78(1.77-4.38)$ & $<0.001$ \\
\hline
\end{tabular}

Note: Numbers are $\mathrm{n}(\%)$ or mean \pm SD unless otherwise indicated.

Abbreviations: MDD, major depressive disorder; OR, odds ratio; $\mathrm{Cl}$, confidence interval; $\mathrm{SD}$, standard deviation.

severity of depression and therefore an increased risk of suicide attempts. Coping skills to deal with stressful life events should be implemented on a routine basis to prevent these tragic acts.

Current use of alcohol was an independent factor associated with a two-fold increase in risk of suicide attempts in our study. Several studies have shown that alcohol use is common among those with depression who attempt suicide. ${ }^{3,6,10,11} \mathrm{We}$ found that $30.2 \%$ of those with MDD used alcohol, which was comparable to the findings of another study conducted in Thailand in which this figure was $33 \% .{ }^{20}$ The explanation for this may be that alcohol causes lower central serotonergic functioning characterized by a propensity towards disinhibited behaviors, therefore increasing aggression and impulsivity and raising the risk of attempting suicide.

Several studies, as well as ours, have shown that previous suicide attempts are the strongest predictive factor of further attempts. ${ }^{5,7,12}$ However, unlike other studies, we classified a 
Table 2 Clinical factors of patients with MDD classified by suicide attempts

\begin{tabular}{|c|c|c|c|c|}
\hline \multirow[t]{2}{*}{ Clinical factors } & \multicolumn{2}{|c|}{ Suicide attempts } & \multirow[t]{2}{*}{ Crude OR $(95 \% \mathrm{Cl})$} & \multirow[t]{2}{*}{$P$-value } \\
\hline & $\begin{array}{l}\text { Yes } \\
(n=186)\end{array}$ & $\begin{array}{l}\text { No } \\
(n=914)\end{array}$ & & \\
\hline \multicolumn{5}{|l|}{ ICD-10 } \\
\hline Mild to moderate & $17(13.4)$ & $110(86.6)$ & 1.00 & \\
\hline Severe & $109(14.7)$ & $632(85.3)$ & $1.12(0.64-1.93)$ & 0.696 \\
\hline With psychosis & $60(25.8)$ & $172(74.1)$ & $2.26(1.25-4.07)$ & 0.007 \\
\hline \multicolumn{5}{|l|}{ Somatic illnesses } \\
\hline No & $121(20.2)$ & $478(79.8)$ & 1.00 & \\
\hline Yes & $57(11.7)$ & $432(88.3)$ & $0.52(0.37-0.73)$ & $<0.001$ \\
\hline \multicolumn{5}{|l|}{ Psychotic comorbidity } \\
\hline No & $139(14.4)$ & $828(85.6)$ & 1.00 & \\
\hline Alcohol/substance dependence & $19(39.6)$ & $29(60.4)$ & $3.90(2.13-7.15)$ & $<0.001$ \\
\hline Others & $9(25.0)$ & $27(75.0)$ & $1.99(0.9|-4.3|)$ & 0.083 \\
\hline \multicolumn{5}{|l|}{ Year(s) of treatment } \\
\hline$<1$ & $152(22.6)$ & $520(77.4)$ & 1.00 & \\
\hline $1-5$ & $12(5.0)$ & $227(95.0)$ & $0.18(0.1-0.33)$ & $<0.001$ \\
\hline$>5$ & $19(11.2)$ & $150(88.0)$ & $0.43(0.26-0.72)$ & 0.001 \\
\hline \multicolumn{5}{|l|}{ Previous admission } \\
\hline No & $136(15.2)$ & $761(84.8)$ & 1.00 & \\
\hline Yes & $48(27.1)$ & $129(72.9)$ & $2.08(1.43-3.04)$ & $<0.001$ \\
\hline \multicolumn{5}{|l|}{ Psychotic symptoms } \\
\hline No & $116(14.0)$ & $711(86.0)$ & 1.00 & \\
\hline Yes & $59(24.7)$ & $180(75.3)$ & $2.00(1.41-2.86)$ & $<0.001$ \\
\hline Mental disorders in family & & & & 0.065 \\
\hline No & $130(17.0)$ & $636(83.0)$ & 1.00 & \\
\hline Yes & $47(15.3)$ & $260(84.7)$ & $0.88(0.6 \mathrm{I}-\mathrm{I} .27)$ & 0.508 \\
\hline \multicolumn{5}{|l|}{ Suicidal history in family } \\
\hline No & $169(16.3)$ & 87I (83.7) & 1.00 & \\
\hline Yes & $14(37.9)$ & $23(62.1)$ & $3.14(1.58-6.22)$ & 0.001 \\
\hline \multicolumn{5}{|l|}{ Previous suicidal attempt } \\
\hline None & $68(8.8)$ & 703 (9।.2) & 1.00 & \\
\hline $\mathrm{I}-2$ times & $77(31.3)$ & $169(68.7)$ & $4.71(3.26-6.80)$ & $<0.001$ \\
\hline$>2$ times & $4 \mathrm{I}(58.6)$ & $29(4 \mid .4)$ & $14.62(8.54-25.00)$ & $<0.001$ \\
\hline \multicolumn{5}{|l|}{ Previous suicidal ideation } \\
\hline No & $57(9.8)$ & $524(90.2)$ & 1.00 & \\
\hline Yes & $129(25.4)$ & $379(74.6)$ & $3.13(2.23-4.39)$ & $<0.001$ \\
\hline \multicolumn{5}{|l|}{ Smoking } \\
\hline No & I4I (I5.7) & $755(84.3)$ & 1.00 & \\
\hline Yes & $30(20.5)$ & 116 (79.5) & $1.38(0.89-2.15)$ & 0.147 \\
\hline \multicolumn{5}{|l|}{ Alcohol use } \\
\hline No & $116(13.5)$ & $744(86.5)$ & 1.00 & \\
\hline Yes & $55(30.2)$ & I $27(69.8)$ & $2.78(1.91-4.03)$ & $<0.001$ \\
\hline \multicolumn{5}{|l|}{ Any substance abuse } \\
\hline No & $158(15.9)$ & $832(84.1)$ & 1.00 & \\
\hline Yes & $13(25.0)$ & $39(75.0)$ & $1.76(0.92-3.36)$ & 0.090 \\
\hline \multicolumn{5}{|l|}{ Medication adherence } \\
\hline Good or excellent & $50(8.9)$ & $511(91.1)$ & 1.00 & \\
\hline Intermittent or poor & $136(25.2)$ & $403(74.8)$ & $3.45(2.43-4.89)$ & $<0.00$ I \\
\hline \multicolumn{5}{|l|}{ Antidepressants } \\
\hline No & $12(18.5)$ & $53(81.5)$ & 1.00 & \\
\hline NE/SRI & $20(9.2)$ & $196(90.8)$ & $0.45(0.2 \mathrm{I}-0.98)$ & 0.044 \\
\hline SSRI & $54 \mid(18.8)$ & 665 (8I.2) & $1.02(0.53-1.96)$ & 0.946 \\
\hline \multicolumn{5}{|l|}{ Antipsychotics } \\
\hline No & $55(8.6)$ & $582(91.4)$ & 1.00 & \\
\hline Yes & $131(28.3)$ & $332(71.7)$ & $4.18(2.96-5.88)$ & $<0.001$ \\
\hline
\end{tabular}


Table 2 (Continued)

\begin{tabular}{|c|c|c|c|c|}
\hline \multirow[t]{3}{*}{ Clinical factors } & \multicolumn{2}{|c|}{ Suicide attempts } & \multirow[t]{3}{*}{ Crude OR (95\% CI) } & \multirow[t]{3}{*}{$P$-value } \\
\hline & Yes & No & & \\
\hline & $(n=186)$ & $(n=914)$ & & \\
\hline \multicolumn{5}{|l|}{ Anxiolytics } \\
\hline No & $35(13.5)$ & $224(86.5)$ & 1.00 & \\
\hline Benzodiazepine & | |4 (18.4) & $505(81.6)$ & $1.44(0.96-2.18)$ & 0.079 \\
\hline Others & $16(12.8)$ & $109(87.2)$ & $0.94(0.49-1.77)$ & 0.847 \\
\hline Both & $21(21.6)$ & $76(78.4)$ & I.77 (0.97-3.22) & 0.063 \\
\hline
\end{tabular}

Note: Numbers are $\mathrm{n}(\%)$ or mean \pm SD unless otherwise indicated.

Abbreviations: MDD, major depressive disorder; OR, odds ratio; $\mathrm{Cl}$, confidence interval; SD, standard deviation; $\mathrm{NE}$, norepinephrine; SRI, serotonin reuptake inhibitor; SSRI, selective serotonin reuptake inhibitor.

continuous number of previous suicide attempts into three groups by intensity, according to degree of OR: none, up to two attempts $(\mathrm{OR}=3.6)$, and more than two attempts $(\mathrm{OR}=11.5)$. As the number of previous suicide attempts increased, the probability of future suicide attempts became substantially higher. Deliberation over more suicidal behaviors reflects greater disease severity and a greater lethality of suicide attempts.

Individuals who had received less than a year of MDD treatment were most vulnerable to suicide attempts. This finding corresponded to a previous study that showed that the

Table 3 Logistic regression coefficient, adjusted OR and 95\% Cl of factors for suicide attempts in individuals with MDD

\begin{tabular}{|c|c|c|}
\hline Factors & $\begin{array}{l}\text { Adjusted OR } \\
(95 \% \mathrm{Cl})\end{array}$ & $P$-value \\
\hline \multicolumn{3}{|l|}{ Stressful life events } \\
\hline No & 1.00 & \\
\hline Yes & $2.32(1.27-4.24)$ & 0.006 \\
\hline \multicolumn{3}{|l|}{ Alcohol use } \\
\hline No & 1.00 & \\
\hline Yes & $2.08(1.29-3.34)$ & 0.003 \\
\hline \multicolumn{3}{|l|}{ Year(s) of treatment } \\
\hline$<1$ & 1.00 & \\
\hline $\mathrm{I}-5$ & $0.22(0.11-0.44)$ & $<0.001$ \\
\hline$>5$ & $0.44(0.23-0.86)$ & 0.016 \\
\hline \multicolumn{3}{|l|}{ Previous suicidal attempt } \\
\hline None & 1.00 & \\
\hline $\mathrm{I}-2$ times & $3.64(2.32-5.7 I)$ & $<0.001$ \\
\hline$>2$ times & I I.47 (5.73-22.95) & $<0.001$ \\
\hline \multicolumn{3}{|l|}{ Medication adherence } \\
\hline Good or excellent & 1.00 & \\
\hline Intermittent or poor & $2.25(I .44-3.5 I)$ & $<0.001$ \\
\hline \multicolumn{3}{|l|}{ Antidepressants } \\
\hline No & 1.00 & \\
\hline NE/SRI & $0.28(0.10-0.78)$ & 0.015 \\
\hline SSRI & $0.85(0.36-1.99)$ & 0.709 \\
\hline \multicolumn{3}{|l|}{ Antipsychotics } \\
\hline No & 1.00 & \\
\hline Yes & $3.84(2.48-5.95)$ & $<0.001$ \\
\hline
\end{tabular}

Abbreviations: MDD, major depressive disorder; $\mathrm{Cl}$, confidence interval; $\mathrm{OR}$, odds ratio; NE/SRI, norepinephrine and/or serotonin reuptake inhibitors; SSRI, selective serotonin reuptake inhibitors. period in which risk of attempted suicide is highest is in the first 3 months after the onset of a major depressive episode. ${ }^{8}$ In addition, a recent study revealed that most suicidal events occurred in the first 6 months after treatment initiation. ${ }^{21}$ It is possible that the establishment of a sustained program of treatment in MDD can be delayed because of the slow onset of some antidepressant medications. This evidence highlighted the need to pay attention to, and be vigilant in, the monitoring of suicide risk in individuals with MDD, particularly during their first year of treatment.

Intermittent or poor adherence to psychiatric medication increased in those for whom more than two previous suicide attempts had been made. In previous studies, inadequacy or suboptimal treatment of depression is one of the reasons that have been given to explain suicide attempts. ${ }^{4,22}$ Good medication adherence contributes to a reduction in suicide attempts by prolonging MDD remission. This finding underlines the importance of optimal maintenance antidepressant treatment as a suicide prevention strategy. Pharmacists should closely monitor and routinely assess the medication adherence of their patients.

In the present study, use of antidepressants was a protective factor against suicide attempts, especially NE/SRIs. The association between SSRIs and suicide attempts was statistically insignificant, but a tendency towards a protective effect was discovered. There has been controversy regarding the possible link between antidepressants and suicidal behaviors. A meta-analysis conducted using all available randomized trials of antidepressants found that the younger the individual, particularly adults aged under 25 years, the greater the risk of suicide attempts. ${ }^{23}$ However, these findings should be cautiously interpreted due to various confounding factors in younger patients, that is, alcohol or illicit substance abuse, impulsivity, and aggressiveness. The inverse relationship of antidepressant use in our study was possibly due to an older MDD population, having an average age of 44.4 years, who obtained greater benefit from this treatment. 
A greater risk of suicide attempts in patients prescribed with antipsychotics was demonstrated, although adherence and antidepressants used were controlled for. The ability to limit risks of suicidal behaviors among those with MDD who have been prescribed antipsychotics remains untested. Clozapine is the only antipsychotic reported to reduce risk of suicidal behaviors, but this is in those with schizophrenia. ${ }^{24}$ We did an additional analysis for a relationship between clozapine use and suicide attempts and revealed no association $(P=0.563)$. The positive relationship of antipsychotics may be confounded by indication and severity of MDD.

Factors such as education, ${ }^{6}$ family history of suicide, ${ }^{14}$ and social support and occupational functioning, ${ }^{3,5}$ have been associated with suicide attempts in previous studies but not in our study. The inconsistency of these findings may be due to discrepancies in population, methodology, assessment tools, and statistical analyses. In addition, some factors were only significant in univariable analysis. Multicollinearity of one or more factors, such as social support and living status, suicidal ideation and previous suicide attempts, may be an explanation.

The present study has some limitations. Firstly, the generalizability of the results may be limited because the study was confined to a tertiary referral psychiatric hospital. Attempted suicide cases sent to a general hospital may be more severely injured. In contrast, those with less severe illness who were not referred to Suanprung Psychiatric Hospital may have used different methods or have a different course of illness. Secondly, retrospective data collection meant that the some of the data were incomplete or missing completely. However, the MI analysis that was performed ensures the final model by giving the same results with a slightly different effect from the complete case analysis. Therefore, we reported results from a complete dataset. Thirdly, some clinical factors, such as hopelessness, impulsiveness, aggression, and hostility score, were not assessed at the study site by instruments used in other studies. Therefore, the ability to detect their potential as risk factors was limited. Finally, the temporal sequence of some variables may be ambiguous due to the nature of the study design, for example, the aforementioned relationship between prescribed medication and severity of disease. Individuals prescribed with more groups of medication had a higher proportion of suicide attempts: $10.0 \%, 11.2 \%$, and $32.2 \%$ in one, two, and three groups of psychiatric medication prescribed, respectively.

In spite of these limitations, the present study provided potential risk factors for suicide attempts among individuals with MDD, and, to our knowledge, this is the first study of its kind in the region in which it was conducted. In the future, prospective research should be undertaken to test for the validity of the factors proposed. Other useful studies recommended are those to assess risk factors predicting completed suicide or first suicide attempts in MDD.

\section{Conclusion}

Suicide attempts were more likely to occur in individuals with MDD who had stressful life events, alcohol use, intermittent or poor medication adherence, previously attempted suicide, and had been prescribed antipsychotics, while increasing years of MDD treatment, and being prescribed antidepressants, particular NE and/or SRIs showed a protective effect. The findings may help in clinician identification of individuals with MDD who are at risk of suicide attempts and provide them close monitoring, timely assessment, and intensive treatments.

\section{Acknowledgments}

This study was in part financially supported by The Faculty of Medicine and The Graduate School, Chiang Mai University.

\section{Disclosure}

The authors report no conflicts of interest in this research.

\section{References}

1. Murray CJ, Lopez AD. Alternative projections of mortality and disability by cause 1990-2020: Global Burden of Disease Study. Lancet. 1997;349(9064):1498-1504.

2. Simon RI, Hales RE, editors. Suicide Risk: Assessing the Unpredictable. 1st ed. Arlington,VA: American Psychiatric Publishing, Inc; 2006.

3. Sokero TP, Melartin TK, Rytsälä HJ, et al. Suicidal ideation and attempts among psychiatric patients with major depressive disorder. J Clin Psychiatry. 2003;64(9):1094-1100.

4. Oquendo MA, Kamali M, Ellis SP, et al. Adequacy of antidepressant treatment after discharge and the occurrence of suicidal acts in major depression. Am J Psychiatry. 2002;159(10):1746-1751.

5. Sokero TP, Melartin TK, Rytsälä HJ, et al. Prospective study of risk factors for attempted suicide among patients with DSM-IV major depressive disorder. Br J Psychiatry. 2005;186:314-318.

6. Suppapitiporn $\mathrm{S}$. The predictors of suicidal attempt in depressed patients. Chulalongkorn University; 2002 [cited November 26, 2009]; Thesis (MSc) - Chulalongkorn University, 2002]. Available from: http://cuir. car.chula.ac.th/dspace/handle/123456789/6093.

7. Holma KM, Melartin TK, Haukka J, Holma IA, Sokero TP, Isometsa ET. Incidence and predictors of suicide attempts in DSM-IV major depressive disorder: a five-year prospective study. Am J Psychiatry. 2010;167(7):801-808.

8. Malone KM, Haas GL, Sweeney JA, Mann JJ. Major depression and the risk of attempted suicide. J Affect Disord. 1995;34(3):173-185.

9. Malone KM, Waternaux C, Haas GL, Cooper TB, Li S, Mann JJ. Cigarette smoking, suicidal behavior, and serotonin function in major psychiatric disorders. Am J Psychiatry. 2003;160(4):773-779. 
10. Sokero TP, Melartin TK, Rytsälä HJ, Leskela US, Lestela-Mielonen PS, Isometsa ET. Prospective study of risk factors for attempted suicide among patients with DSM-IV major depressive disorder. $\mathrm{Br} J$ Psychiatry. 2005;186:314-318.

11. Chan LF, Maniam T, Shamsul AS. Suicide attempts among depressed inpatients with depressive disorder in a Malaysian sample. Psychosocial and clinical risk factors. Crisis. 2011;32(5):283-287.

12. Oquendo MA, Currier D, Mann JJ. Prospective studies of suicidal behavior in major depressive and bipolar disorders: what is the evidence for predictive risk factors? Acta Psychiatr Scand. 2006;114(3): 151-158.

13. Van Gastel A, Schotte C, Maes M. The prediction of suicidal intent in depressed patients. Acta Psychiatr Scand. 1997;96(4):254-259.

14. Mann JJ, Waternaux C, Haas GL, Malone KM. Toward a clinical model of suicidal behavior in psychiatric patients. Am J Psychiatry. 1999; 156(2):181-189.

15. Perroud N, Baud P, Mouthon D, et al. Impulsivity, aggression and suicidal behavior in unipolar and bipolar disorders. $J$ Affect Disord. 2011;134(1-3):112-118.

16. Department of Mental Health, Ministry of Public Health Thailand. Suicide Rates: Thailand (per 100,000 population) by provinces (top ten). Bangkok, Thailand 2011 [cited 2012 Feb 15]. Available from: http:// www.dmh.go.th/report/suicide/english/. Accessed February 15, 2012.
17. Suanprung Psychiatric Hospital. Suanprung psychiatric hospital report number of patients between 2009 to 2011. Chiang Mai, Thailand 2011 [cited 2012 Feb 15]. Available from: http://www.suanprung.go.th/eng/ top_five.php. Accessed February 15, 2012.

18. Myers WR. Handling missing data in clinical trials: an overview. Drug Inf J. 2000;34:525-533.

19. Azorin J, Kaladjian A, Besnier N, et al. Suicidal behaviour in a French Cohort of major depressive patients: characteristics of attempters and nonattempters. J Affect Disord. 2010;123(1-3):87-94.

20. Suppapitiporn S. Comorbidity of alcohol dependence in suicidal depressed patients. J Med Assoc Thai. 2005;88 Suppl 4:S195-S199.

21. Schneeweiss S, Patrick AR, Solomon DH, et al. Variation in the risk of suicide attempts and completed suicides by antidepressant agent in adults: a propensity score-adjusted analysis of 9 years' data. Arch Gen Psychiatry. 2010;67(5):497-506.

22. Isacsson G, Bergman U, Rich CL. Epidemiological data suggest antidepressants reduce suicide risk among depressives. J Affect Disord. 1996;41(1):1-8.

23. Stone M, Laughren T, Jones ML, et al. Risk of suicidality in clinical trials of antidepressants in adults: analysis of proprietary data submitted to US Food and Drug Administration. BMJ. 2009;339:b2880.

24. Baldessarini RJ, Pompili M, Tondo L. Suicide in bipolar disorder: risks and management. CNS Spectr. 2006;11(6):465-471.
International Journal of General Medicine

\section{Publish your work in this journal}

The International Journal of General Medicine is an international, peer-reviewed open-access journal that focuses on general and internal medicine, pathogenesis, epidemiology, diagnosis, monitoring and treatment protocols. The journal is characterized by the rapid reporting of reviews, original research and clinical studies across all disease areas.

\section{Dovepress}

A key focus is the elucidation of disease processes and management protocols resulting in improved outcomes for the patient.The manuscript management system is completely online and includes a very quick and fair peer-review system. Visit http://www.dovepress.com/ testimonials.php to read real quotes from published authors. 